\title{
Psychosocial research in spinal cord injury: the state of the art
}

\author{
R B Trieschmann $\mathrm{PhD}$ \\ Consulting Psychologist, Box 5566, Scottsdale, Arizona, 85261, USA.
}

\begin{abstract}
A half century ago, people with SCI were not expected to live and those that did were not expected to function in normal society. Spinal injury was considered to be the ultimate devastation that could occur in one's life, and to survive such an assault was considered to be extraordinarily difficult. Thus, much of the psychosocial research of the 1940 s-1960s focused on describing the extent of the emotional carnage which the investigators assumed was present, and attempts were made to predict who would cope with the disability and who would not. An implicit assumption behind much of the early research was that the SCI itself produced not only major physical consequences but also grave psychological consequences which made these people inherently 'different' from non disabled individuals. Thus, the earlier research examined the psychological characteristics of those with spinal cord injury in order to document these differences. However, as we approach the last decade of the twentieth century, it is clear that people with physical disabilities are no different from non disabled people except for the presence of a physical impairment. Thus, the major research question now is, 'What are the processes of coping with the spinal cord injury?'. ${ }^{1}$
\end{abstract}

Recently, there has been concern that a number of individuals may sustain an associated head injury that has not been detected in the past. However, much of the research on this topic has been methodologically flawed by conducting cognitive testing early in the hospitalization phase when the intellectually obtunding effects of the hospital environment are paramount. Furthermore, many studies do not control for preinjury intellectual function and mood during this crisis stage in one's life. The primary issue to be determined is whether an individual experiences a reduction in intellectual func- tion from preinjury level which compromises personal and vocational activities years after onset of the SCI. It has not been demonstrated that this is the case in a large number of individuals and, thus, methodologically sound research is needed in this area.

For decades professionals believed that people with new spinal cord injuries must get depressed in order for adjustment to occur. However, research demonstrates that most do not get depressed. Grief, yes; depression, no. Thus our semantics, our definitions of the early emotional reactions, and our measurement procedures need to be clarified. Using a classical psychiatric definition of depression, only a very small percentage of newly spinal injured people exhibit major depression. When it does occur, it is usually in the acute treatment phase and tends to resolve within a few weeks or before discharge. Most individuals cannot envisage how they can possibly live a normal life with the physical impairment and are unhappy about this, but this is based upon lack of information as to what is really possible because they are still in the hospital. Psychological test evidence does not reveal depression nor psychological devastation at all in most cases. Rather people tend to focus on coping with the immediate physical reality of the disability while the rehabilitation therapies confront one with the extent of the loss. Actually most people do very well with this since, in actuality, they have very little choice. Thus, the question is, 'How do we teach people to cope more effectively with this disability?'

SCI does, indeed, assault one's personal identity. A whole series of 'I am's' are no longer relevant and an identity vacuum is created initially. The process of coping with the disability involves letting go of some 'I am's' and developing other ones about 
which the person can feel good. This occurs, however, within the context of a family, hospital environment, and society that subtly communicates the message that 'you are less than you used to be'. At all costs, one must strive to be 'normal', and the emotional and physical cost is a considerable one. What are the methods of reducing this cost?

Professionals often worry about the motivation of the person with SCI, but motivation usually does not become an issue until it becomes one of non compliance with recommended procedures. The assumption is made that the professional recommendations are the correct ones and the person should want to cooperate in order to get better. However, evidence demonstrates that hospital environments permit little independence or decision making on the part of the person with the disability. Thus, sometimes the only measure of independence and motivation possible is non compliance. Thus, the research and practice issue becomes, 'How can we facilitate better communication among professionals and people with SCI to enhance independent functioning within the hospital and community environment?'

Integration into the community is a multiyear process with the initial tasks centered on the incorporation of the new activity of daily living and mobility skills as an automatic part of life plus the trial and error period of trying to prevent medical complications. Without a doubt, survival with SCI depends on the acquisition of impeccable self care skills and the ability to tune into body function to anticipate trouble before it becomes a problem. But in order to live, not only survive, the person needs to face a society which frequently sends the message that you do not belong (through inaccessible environments), that you are different (through anxiety or avoidance by non disabled people in social situations), and that you are now a second class citizen (through a program of financial disincentives which penalize attempts to become productive and financially self sufficient). How do we teach people to cope with these environmental barriers? This is the focus for research.
Despite these obstacles, many people manage to cope very well and create a reasonable life for themselves. However, spinal cord injury 'occurs' to the family members as well and stress is introduced by the changes which the disability imposes on the entire family constellation. Pace of life slows down, income must be allocated differently, social activities change, and therefore effective communication is the key to maintaining a viable family unit. Research shows that the patterns of marital stress are no different for non disabled and disabled couples, but the content areas of the stress may be slightly different. The ability to be a good parent is not challenged by the onset of a physical disability; in fact research demonstrates that children of parents with disabilities are more sensitive and caring toward their parents than children of non disabled individuals. Thus an important set of 'I am's' to be developed after SCI is in the area of relationships: 'I am a lover; I am a husband or wife; I am a mother or father; I am a friend; I am a good person'. The research question again is, 'How do people cope with the altered relationships and what strategies can be used to facilitate a harmonious set of 'I am's'?'

The fight for financial survival adds to the stress of the physical and emotional penalty of SCI, and evidence from our long term survivors suggests that this stress may take its toll in terms of earlier onset of physical decline associated with aging than in a similar aged non disabled population. Therefore, the focus on recreation, athletics, health and wellness programs of our younger generation of SCI seems very appropriate. It provides a rewarding set of 'I am's' to offset the negative messages sent by society regarding employment and is associated with fewer hospitalizations and medical complications than non active SCI. What is the health status of those within a decade or 2 of the SCI? What are the best methods of promoting wellness in the SCI community? How does this influence coping and quality of life?

Since a certain percentage of spinal injuries occur during the use of alcohol or drugs, the question of continued usage of such substances has been raised. If the use 
evolves into abuse in response to the disability, this can compromise function in exceedingly costly ways. Therefore we need to assess the incidence of the problem and develop strategies for dealing with it. Essentially the key is to assist the person to find rewarding 'I am's' after the SCI and to learn other methods of handling stress. This is a critical focus for research in this decade given the increasing usage of mind and mood altering substances by a large portion of our youth. How do we teach coping skills to new SCI with a history of alcohol and drug abuse? Merely counting the incidence of the problem is insufficient. Research must focus on interventions to remedy the problem.

Women with SCI fight the dual minority status of being not only a woman but also disabled. Often she is disenfranchised from long term partnerships because a man's status is often judged by the physical perfection of his mate. Yet we know little about the coping ability of women with severe disability because they are infrequently the subject of research. Clinical observation reveals that women, in contrast to their frail and helpless image, are exceedingly strong and have significant resources to cope despite the lack of interpersonal support which many men have. Thus, the coping style of women with SCI is a much neglected but critically needed area for future research.

In essence we can say that a normal life is decidedly possible after onset of SCI. Nevertheless, no matter how good the resources of the individual and the environment are, living with a severe disability takes its toll emotionally and physically. Yet this toll is rarely discussed with family, friends, or professionals. The need to maintain face, to look good, to be capable, to be in control, both emotionally and physically, becomes so deeply ingrained that often the person loses touch with his or her own feelings. They are suppressed and repressed so continuously that the person may not be aware of the amount of anger, frustration, and fatigue that have accumulated. Depression is not a frequent occurrence but anxiety about the future with increasing age certainly is.

Thus, a recent phenomenon is the arrival into seniority of a group of people with SCI who have lived with the disability for 30,40 , and 50 years. ${ }^{2}$ They represent the crème de la crème of adjustment to disability. They are survivors, self sufficient, and exceedingly capable of understanding what their bodies need to survive. At this point, however, they seem to be experiencing a constellation of symptoms which varies across individuals and which seems to represent an early aging process. This is adding to the physical penalty of the disability which impacts on the person's self image and environmental resources. The health care system is quite unprepared to treat older people who have lived many years with a physical disability, since the average community practitioner knows little about rehabilitation . Furthermore, the rehabilitation community has focused almost exclusively on the acute management of disability and now is unprepared to provide services to this generation of survivors. Consequently, we need a program of research and service delivery which focuses on creating a partnership between the professional and the person with the disability, one which acknowledges the expertise of the SCI in managing his or her own body for all of these years. This, too, must become a major focus for the 1990s.

\section{References}

1 Trieschmann RB (1988) Spinal Cord Injuries: Psychological, Social, and Vocational Rehabilitation. Demos Publications, New York.

2 Trieschmann RB (1987) Aging with a Disability. Demos Publications, New York. 